\title{
Improving RF Safety in MRI by Modifying the Electric Field Distribution
}

\author{
Yigitcan Eryaman $^{1}, \underline{\text { Ergin Atalar }}^{1}$ \\ ${ }^{1}$ National Magnetic Resonance Research Center (UMRAM), Department of Electrical and Electronics Engineering, \\ Bilkent University,Ankara, Turkey
}

\begin{abstract}
In this work we demonstrate that the radiofrequency (RF) electric field in magnetic resonance imaging (MRI) can be modified in order to enhance patient safety. The heating of metallic devices in MRI is directly related to electric field distribution. On the other hand the MR image homogeneity is related to forward polarized component of the magnetic field (transmit sensitivity). In order to prevent heating, electric field-free zones should be generated in the body without significantly altering the transmit sensitivity. For this purpose the linearly polarized birdcage coil is proposed as a metallic device friendly MRI coil. The zero electric field plane of the linear birdcage coil is coincided with the location of the metallic device and the heating is reduced as shown by simulations and experiments. One disadvantage of this approach is, the linear coils generate twice as much whole body average SAR when compared to quadrature birdcage coils. In order to solve this problem simulations are performed to find electromagnetic field solutions with reduced average SAR and uniform transmit sensitivity.
\end{abstract}

\section{Introduction}

MRI is a safe imaging technology which enables visualization of the soft tissue inside the human body. For patients who carry metallic devices such as implants, there exists a risk of excessive heating of the tissue in the vicinity of the metallic device lead [1]. This risk has been investigated both theoretically and experimentally in previous works [2]. The common approach to this problem is to modify the metallic device and make it MRcompatible so that the device does not exhibit any RF heating during MRI. This modification is usually made by adding lump elements to the lead extension of the device which has the maximum potential for heating. In one of the studies a series of RF chokes were added to reduce the induced currents on the shield of the cable[3]. Alternative geometries and solutions have been proposed as well. [4] All of these solutions require modifying the metallic device electrically to reduce RF heating. However this solution may not always be feasible. First of all, for patients who already have non MR-compatible implants in their body, it is not practical to change the implant with the safer one in order to get an MR scan. Furthermore for certain interventional procedures involving simple metallic devices such as biopsy needles [5] it is very difficult to make electrical modification on the device. For this purpose a different solution was proposed in an earlier work which focuses on the EM field distribution in the vicinity of the device [6]. In that work the EM field distribution in the body was optimized to generate electric field-free zones and reduce RF heating of metallic devices. The linearly polarized birdcage coil was proposed as an implementation of such a field distribution. The linearly polarized birdcage excitation caused twice as much SAR as compared to conventional quadrature excitation. To alleviate this problem, the field solutions were optimized to reduce average SAR and obtain uniform transmit sensitivity. In this work we present a summary of these results.

\section{Theory}

The electric field due to quadrature and linear RF excitation can be approximated as follows:

$$
E_{z}=\frac{-H_{f} \omega \mu_{0} \rho}{2} e^{j \phi}, E_{\phi}=0, E_{\rho}=0
$$

$$
E_{z}=-H_{f} j \omega \mu_{0} \rho \sin \phi, E_{\phi}=0, E_{\rho}=0
$$

In the above expressions, an infinitely long birdcage coil is assumed for excitation. Similarly, body is assumed as an infinitely long cylinder with uniform electromagnetic properties. $H_{f}$ is the transmit sensitivity of the coil and $E_{z}, E_{\phi}, E_{\rho}$ are the longitudinal, angular and radial components of the electric field, respectively; $\omega$ is the 
Larmor frequency; $\mu_{0}$ is the uniform permeability; $\rho$ and $\phi$ are the radial and angular coordinates in the cylindrical coordinate system, respectively; $j$ is the imaginary number defined as $\sqrt{-1}$. On the other hand the electric field due to linear excitation can be approximated as follows:

As it can be noted from the above equations, the electric field of the linearly polarized birdcage coil is equal to zero over the entire $\phi=0$ plane. In general the zero electric field plane can be steered into any angular direction either by changing the feeding or rotating the coil around the $\mathrm{z}$ axis. If the metallic device is confined in a cylindrical plane inside the phantom, the coil can be rotated in order to coincide the zero electric field plane with the location of the device. In that condition there will be no induced currents on the device and the local heating problem will be reduced.

The linear birdcage coils solve the problem for RF heating of metallic devices in MRI. However they cause twice as much SAR as a conventional quadrature birdcage coil. In order to solve this problem a general formulation is developed. The electric field and the transmit sensitivity inside a homogenous uniform phantom can be expressed as an infinite summation of cylindrical modes as shown below: Transmit sensitivity is equal to forward polarized magnetic field component of the magnetic field.

$\vec{E}=\sum_{m=-\infty}^{\infty} \sum_{n=-\infty}^{\infty} \boldsymbol{E}_{\boldsymbol{m} n} \cdot \vec{\alpha}_{m n} \cdot e^{j m \phi} e^{-j \beta_{z} z}$

$$
H_{f}(\vec{r})=\sum_{m n} \vec{H}_{f m n}(\vec{r}) \cdot \vec{\alpha}_{m n} \cdot e^{j(m+1) \phi} e^{-j \beta_{z n} z}
$$

Here $\mathrm{m}$ and $\mathrm{n}$ are the index of the cylindrical modes used in the basis expansion. $\phi$ and $\mathrm{z}$ are the angular and $\mathrm{z}$ coordinates in the cylindrical coordinate system, respectively. $\boldsymbol{E}_{\boldsymbol{m} \boldsymbol{n}}$ is a $3 \times 2$ matrix that contains the electric field basis functions for the $\rho, \phi$ and z components $\vec{\alpha}_{m n}$ is a $2 \mathrm{x} 1$ vector whose elements are the constants that multiply the basis functions and $\vec{\alpha}_{m n}=\left[A_{m n} B_{m n}\right]^{T}$.

In order to obtain uniform transmit sensitivity, the forward polarized magnetic field should be constrained to unity at desired locations in the body. On the other hand, to handle the local heating problem the electric field component which is parallel to the lead of the metallic device should be set to zero. These conditions can also be expressed as a linear equation as follows:

$$
E \alpha=\overrightarrow{0}
$$

$$
H \alpha=c
$$

The elements of $H$ are equal to basis functions of $H_{f}(\vec{r})$ evaluated at the desired sample points. $\mathrm{c}$ is a vector whose elements are equal to unity. $\alpha$ is a vector whose elements are equal to weighting coefficients, $\left(A_{m n} B_{m n}\right)$.

To handle the local heating problem the electric field component which is parallel to the lead of the metallic device should be set to zero. Similar to equation 5 this condition can also be expressed as a linear equation as follows:

The constraints on transmit sensitivity and the electric field equations 5 and 6 can be combined as in the following expression, $F \alpha=e$.

In order to improve the RF safety the average SAR should also be reduced. The expression for the average SAR is shown in equation 6.

$$
\mathrm{SAR}=\sigma / M \int_{b o d y}|E|^{2} d v
$$

In this equation $\sigma$ is the conductivity and $\mathrm{M}$ is the total body mass, $\mathrm{dv}$ is the differential volume element. The average SAR can be expressed as a quadratic function of $\alpha$ as $\alpha^{*} R \alpha$, where $\mathrm{R}$ is the electric field cross correlation. For a detailed derivation of the $\mathrm{R}$ matrix, the previous publication [6] can be reffered.

There exist infinitely many different $\alpha$ that satisfy $F \alpha=e$. Each $\alpha$ corresponds to a different field distribution in space. Among these infinitely many different field distributions the one that results in minimum average SAR can be chosen as the optimum solution. For this purpose $\alpha^{*} R \alpha$ should be minimized with the linear constraint, $F \alpha=e$. The solution for this problem is shown in equation 7 and 8 .

$$
\alpha_{o p t}=R^{-1} F^{*}\left(F R^{-1} F^{*}\right)^{-1} e
$$

$$
S A R_{\min }=e^{*}\left(F R^{-1} F^{*}\right)^{-1} e
$$




\section{Simulations and Experiments}

To verify the theory, phantom experiments are performed. The phantom head model $16 \mathrm{~cm}$ in diameter and $25 \mathrm{~cm}$ in length is prepared with commercially available gel (Dr Oetker Jello, Izmir, Turkey). $2.4 \mathrm{~g} / 1 \mathrm{salt}$ is added to the gel solution to obtain a conductivity of $0.51 \mathrm{~S} / \mathrm{m}$ and relative permittivity of 70 . Metallic bare wires are placed inside phantoms as shown in Figure 1. Then the phantoms are scanned by using a gradient echo (GRE) sequence and exposing the phantoms to maximum local SAR of $4.4 \mathrm{~W} / \mathrm{kg}$. The temperature variation at the tip of the wires are measured with fiber optic temperature probes(Neoptix Inc, Quebec City, Canada). Quadrature and linear excitation is used to scan both the straight and curved wires. To obtain linear excitation one of the ports of the coil was disconnected. Then the coil is rotated to coincide the location of the wire with the zero electric field plane of the coil. The phantom is then scanned and the temperature data regarding the minimum heating condition is measured. Then the phantom is rotated 90 degrees and the phantom is scanned once more using the maximum heating condition. Finally the phantom is scanned with quadrature excitation.

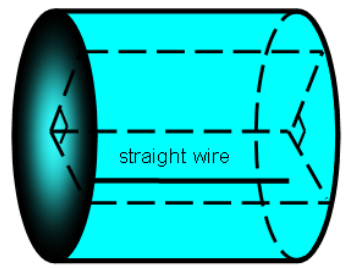

a

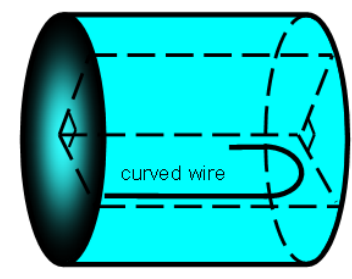

d

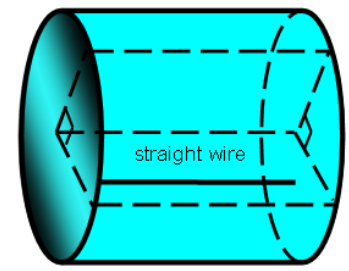

b

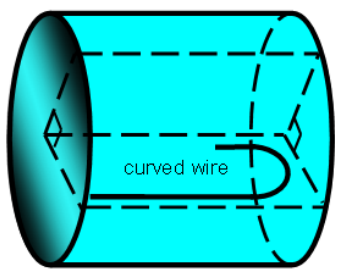

e
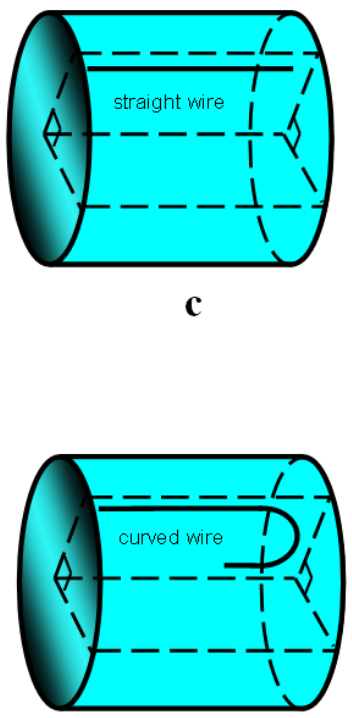

f

Figure 1. Gel phantoms with straight and curved wires. Fiber-optic temperature measurements were performed near the tips of the lead wires. (a) and (b), quadrature excitation; (c) and (d), linear excitation under the minimum heating condition; (e) and (f), linear excitation under the maximum heating condition.

The linear birdcage coils can be used to reduce the RF heating of metallic devices. However they cause twice as much SAR as compared to quadrature birdcage coils. To solve this problem EM field inside the phantom model is optimized. Field solutions regarding 4 different optimum coils are calculated; First the field distribution of the optimum Quadrature coil is calculated. For this purpose a single constraint is imposed on the transmit sensitivity. Second, the field of the optimum linear coil is calculated. The conjugates of the expansion coefficients are used to construct the field distribution of the optimum linear coil. Third the field of the implant friendly coil is calculated. The transmit sensitivity of the optimum linear coil is used as a constraint for this solution. Additionally the electric field on a hypothetical straight wire inside a phantom is set to zero. Finally the field of the homogenous implant friendly coil is calculated. In addition to electric field constraints used for the implant friendly coil, transmit sensitivity is set to unity in the entire transverse plane. By doing so a perfectly homogenous transmit sensitivity was obtained. In all of the 4 solutions, average SAR is minimized.

\section{Results}

Maximum temperature increases of $0.8^{\circ} \mathrm{C}, 24.7^{\circ} \mathrm{C}, 12.1^{\circ} \mathrm{C}$ were observed at the tip of the straight lead with 
minimum heating linear mode, maximum heating linear mode, and quadrature mode respectively. For the curved wire, maximum temperature increases of $0.3^{\circ} \mathrm{C}, 19.1^{\circ} \mathrm{C}$ and $9.2^{\circ} \mathrm{C}$ were observed with the same excitation modes.

As the measurements imply, the RF heating of metallic leads and wires can be reduced by using a linear birdcage coil. The transmit sensitivity and the electric field distribution of the 4 optimum field solutions are also calculated. Figure 2 shows the field distributions.

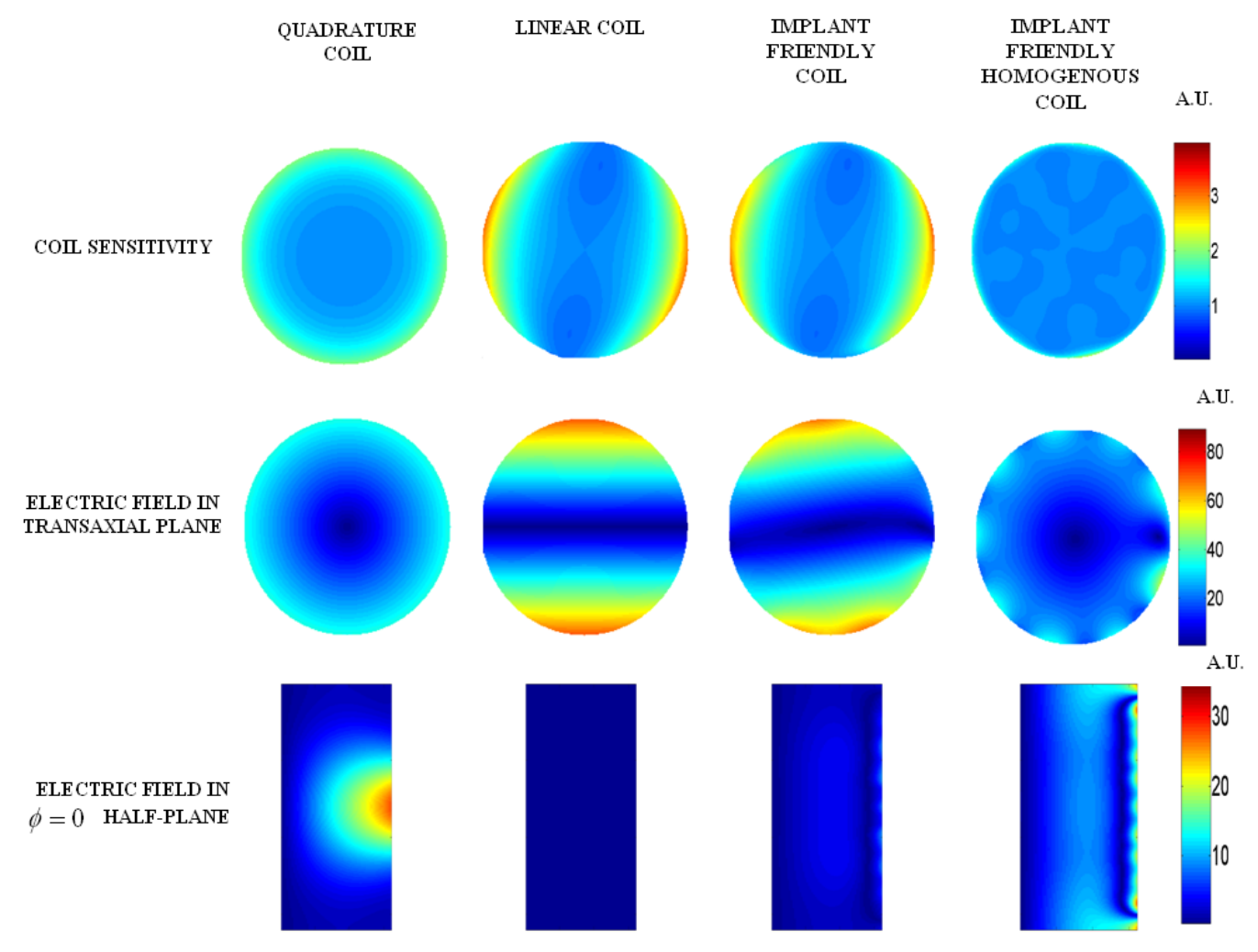

Figure 2. Transmit sensitivity, electric field in the trans-axial plane and electric field in the $\phi=0$ half-plane generated by the optimum quadrature, linear, implant-friendly and implant-friendly homogeneous coils are shown.

\section{Conclusions}

In this study, we demonstrated that RF safety in MRI can be improved by modifying the electric field distribution. The linear birdcage coil is used to reduce the RF heating of metallic wires. The electric field in the vicinity of a metallic device and the whole body average SAR can be minimized while obtaining a desired transmit sensitivity.

\section{References}

[1] Henderson J. Permanent neurological deficit related to magnetic resonance imaging in a patient with implanted deep brain stimulation electrodes for Parkinson. Neurosurgey 2005:57

[2]Yeung, C. J., R. C. Susil, et al. (2002). "RF safety of wires in interventional MRI: Using a safety index." Magnetic Resonance in Medicine 47(1): 187-193.

[3] Ladd ME, Quick HH. Reduction of resonant RF heating in intravascular catheters using coaxial chokes. Magn Reson Med 2000;43(4):615-619.

[4] Bottomley PA,Kumar A,Edelstein WA,Allen JM,Karmarkar PV. Designing passive MRI-safe implantable conducting leads with electrodes. Med Phys 2010;37(7):3828-43

[5]Krieger, A., R. C. Susil, et al. (2005). "Design of a novel MRI compatible manipulator for image guided prostate interventions." IEEE Transactions on Biomedical Engineering 52(2): 306-313.

[6]Eryaman Y, Akin B. Atalar E. Reduction of implant RF heating through modification of electric field Mag Reson Med 2010 n/a. doi: 10.1002/mrm.22724 